\title{
ASPECTS OF THE QUANTUM CHROMO DYNAMICS PHASE DIAGRAM
}

\author{
FRANCESCO SANNINO \\ NORDITA \\ Blegdamsvej 17, Copenhagen Ø, DK-2100 Denmark \\ E-mail: francesco.sannino@nbi.dk
}

\begin{abstract}
I briefly review some aspects of the Quantum Chromo Dynamics phase diagram at non zero temperature and quark chemical potential. I then suggest new possible phases which can appear in strongly interacting theories at non zero chemical potential. Finally I describe the phase diagram as function of the number of flavors and colors at zero quark chemical potential and zero temperature.
\end{abstract}

\section{Introduction}

There are already many excellent reviews on the QCD phasediagram, its possible applications and effective theories approaches 1. first introduce some basic features of the phase diagram and then suggest new phases which can affect it such as vectorial-type condensation. I will also provide a brief summary of a possible plan for the phase diagram when varying the number of light flavors relative to the number of colors but keeping zero temperature and quark chemical potential and motivate the relevance for the physics beyond the standard model of particle interactions.

\section{The Hot and Dense QCD Phase Diagram}

\subsection{Hadronic/Confining Phase}

At zero temperature and density we do not observe free quarks and gluons. These states are permanently confined in hadronic particles like pions, vectors, nucleons etc. In this regime the interactions among hadrons can be efficiently described via effective Lagrangians built respecting the relevant symmetries of the underlying theory.

\subsection{Hot and dilute $Q C D$}

At temperatures just above the deconfinement phase transition but small quark chemical potential the theory enters a quark gluon plasma phase (QGP) in which the underlying states are still strongly interacting but deconfined. Only at asymptotically high temperatures perturbative QCD calculations are

QCDPD: submitted to World Scientific on November 16, 2018 


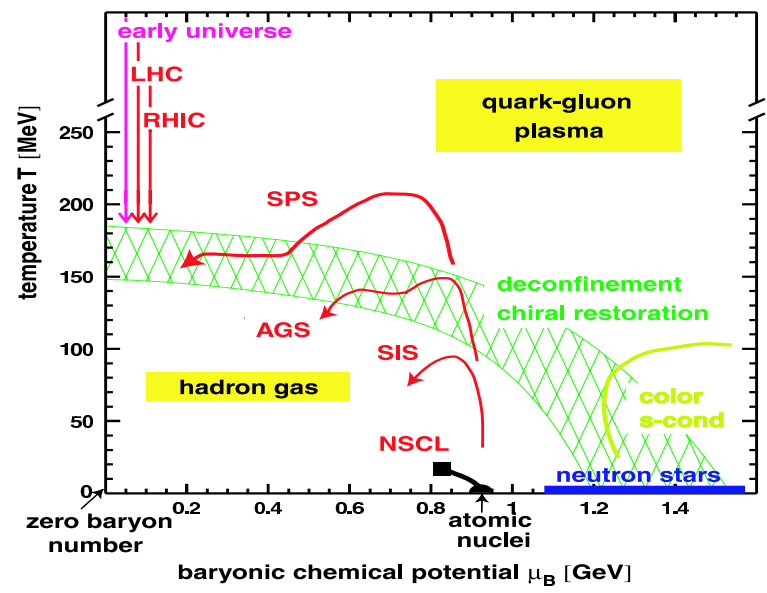

Figure 1. Possible physical applications and region of the QCD phase diagram explored by different experiments.

really reliable. This phase is relevant for the physics of the early universe and is accessible to earth based experiments like RHIC and LHC. Figure 1 l shows the relevant experiments and possible physical applications.

The study of the temperature driven confining-deconfining phase transition has attracted much interest and work. Thanks to lattice simulations we now have a great deal of information. More specifically all of the relevant thermodynamical quantities have been computed with and without quarks 0.10 at zero quark chemical potential. Very recently new methods have been proposed which might help studying the phase diagram at non zero chemical potential via lattice simulations 11 . However still much is left to be understood about the nature of the transition of hot hadronic matter to a plasma of deconfined quarks and gluons $\mathrm{B}$.

\section{Dense and Cold QCD: Color Superconductivity}

At zero temperature but very high quark chemical potential the perturbative ("naive") vacuum is unstable. In this regime strong interactions favor the 
formation of quark-quark condensates in the color antisymmetric channel 1 . Possible physical applications are related to the physics of compact objects 1 , supernprae cooling 12 and explosions 13 as well as to the Gamma Ray Bursts puzzle 14.

The color superconductive phase is characterized by its gap energy $(\Delta)$ associated to quark-quark pairing which leads to the spontaneous breaking of the color symmetry. According to the number of light flavors in play we can have different phases.

\subsection{Color Flavor Locked Phase}

Let us start with the case of $N_{f}=3$ light flavors. At zero density only the confined Goldstone phase is allowed and the resulting symmetry group is $S U_{V}(3) \times U_{V}(1)$.

Turning on low baryon chemical potential we expect the theory to remain in the confined phase with the same number of Goldstone bosons (i.e. 8). At very high chemical potential, dynamical computations suggest that the preferred phase is a superconductive one and the following ansatz for a quarkquark type of condensate is energetically favored:

$$
\epsilon^{\alpha \beta}<q_{L \alpha ; a, i} q_{L \beta ; b, j}>\sim k_{1} \delta_{a i} \delta_{b j}+k_{2} \delta_{a j} \delta_{b i} .
$$

A similar expression holds for the right transforming fields. The Greek indices represent spin, $a$ and $b$ denote color while $i$ and $j$ indicate flavor. The condensate breaks the gauge group completely while locking the left/right transformations with color. The final global symmetry group is $S U_{c+L+R}(3)$, and the low energy spectrum consists of 9 Goldstone bosons.

The low energy effective theory for 3 flavors (CFL) has been developed in 15. We refer to 1.6 for a complete summary and review of this phase.

\subsection{SC 83 Partial Deconfinement}

QCD with 2 massless flavors has gauge symmetry $S U_{c}(3)$ and global symmetry

$$
S U_{L}(2) \times S U_{R}(2) \times U_{V}(1) .
$$

At very low baryon chemical potential it is reasonable to expect that the confined Goldstone phase persists. However at very high density the ordinary Goldstone phase is no longer favored compared with a superconductive one associated to the following type of diquark condensates:

$$
\left\langle L^{\dagger^{a}}\right\rangle \sim\left\langle\epsilon^{a b c} \epsilon^{i j} q_{L b, i}^{\alpha} q_{L c, j ; \alpha}\right\rangle, \quad\left\langle R^{\dagger^{a}}\right\rangle \sim-\left\langle\epsilon^{a b c} \epsilon^{i j} q_{R b, i ; \dot{\alpha}} q_{R c, j}^{\dot{\alpha}}\right\rangle
$$

QCDPD: submitted to World Scientific on November 16, 2018 
$q_{L c, i ; \alpha}, q_{R c, i}^{\dot{\alpha}}$ are respectively the two component left and right spinors. $\alpha, \dot{\alpha}=$ 1,2 are spin indices, $c=1,2,3$ stands for color while $i=1,2$ represents the flavor. If parity is not broken spontaneously, we have $\left\langle L_{a}\right\rangle=\left\langle R_{a}\right\rangle=f \delta_{a}^{3}$, where we choose the condensate to be in the 3rd direction of color. The order parameters are singlets under the $S U_{L}(2) \times S U_{R}(2)$ flavor transformations while possessing baryon charge $\frac{2}{3}$. The vev leaves invariant the following symmetry group:

$$
\left[S U_{c}(2)\right] \times S U_{L}(2) \times S U_{R}(2) \times \widetilde{U}_{V}(1)
$$

where $\left[S U_{c}(2)\right]$ is the unbroken part of the gauge group. The $\widetilde{U}_{V}(1)$ generator is linear combination of the previous $U_{V}(1)$ generator and the broken diagonal generator of the $S U_{c}(3)$ gauge group $T^{8}$. The quarks with color 1 and 2 are neutral under $\widetilde{U}_{V}(1)$ and consequently the condensate is neutral too.

The superconductive phase for $N_{f}=2$ possesses the same global symmetry group as the confined Wigner-Weyl phase 16 . The effective theory for 2SC can be found in 17 while the effective theories describing the electroweak interactions for the low-energy excitations in the 2SC and CFL case can be found in 18. The global anomalies matching conditions for 2 and 3 flavors and constraint are discussed in 16.

An interesting property of the $2 S C$ state is that the three color gauge group breaks via a dynamical higgs mechanism to a left over $S U_{c}(2)$ subgroup. In Reference 19 it has been shown that the confining scale of the unbroken $S U_{c}(2)$ color subgroup is lighter than the superconductive gap $\Delta$. The confined degrees of freedom, glueball-like particles, are expected to be light with respect to $\Delta$, and the effective theory based on the anomalous variation of the dilation current has been constructed in 20 Using this model Lagrangian extended to include non zero temperature in 21 the following simple expression for the $S U_{c}(2)$ critical temperature was found:

$$
T_{c}=\sqrt[4]{\frac{90 v^{3}}{2 e \pi^{2}}} \hat{\Lambda} .
$$

Here $e$ is the Euler number, $\hat{\Lambda}$ is the confining scale of the $S U_{c}(2)$ gluondynamics in 2SC and $v$ is the gluon 19 as well as light glueball velocity 20 . The deconfining/confining critical temperature is smaller than the critical temperature $T_{2 S C}$ for the superconductive state itself which is estimated to be $T_{2 S C} \approx 0.57 \Delta$ with $\Delta$ the 2SC gap 22 . Knowing the explicit dependence of the $S U_{c}(2)$ confining temperature on $\mu$ and $\Delta$ directly affects astrophysical models for compact stars like the one in Ref. 14. A second order phase transition is also predicted. Finally in 21 it is suggested how ordinary lattice

QCDPD: submitted to World Scientific on November 16, 2018 


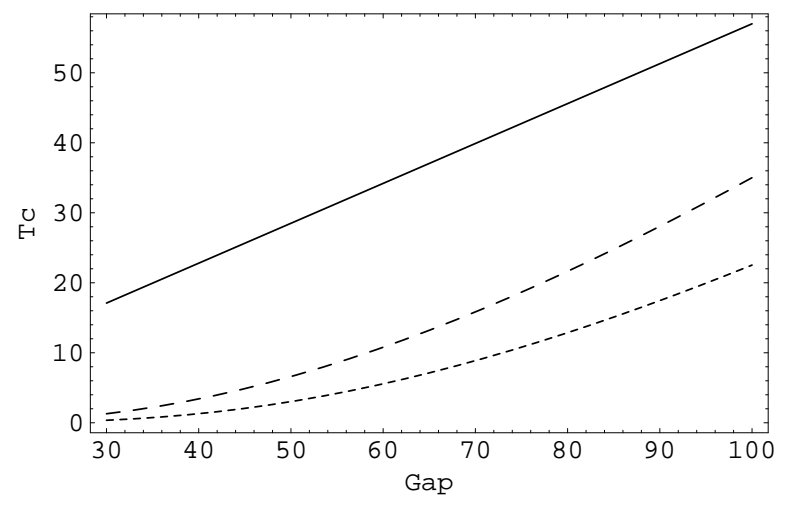

Figure 2. Plot of the $S U_{c}(2)$ critical temperature for 2 values of the quark chemical potential ( $\mu=400 \mathrm{MeV}$ long-dashed line; $\mu=500 \mathrm{MeV}$ short-dashed line) as function of the superconductive gap $\Delta$. The solid line corresponds to the critical temperature for the superconductive state $0.57 \Delta$.

importance sampling techniques can be used to check these results and constitute, at the same time, the first simulations testing the high quark chemical potential but small temperature region of the QCD phase diagram. Interestingly recently it has also been shown that at high chemical potential the effective field theory of lgw energy modes in dense QCD has positive Euclidean path integral measure 23. For temperatures in the range $T_{c}<T<T_{2 S C}$ the gapped quark dynamics is no longer negligible. Using the transport theory sqme of the quark temperature dependent effects have been investigated in 24. It has also been recently argued that a $2 \mathrm{SC}$ phase might not appear in compact stars 25 .

In a world with two light and approximately degenerate quarks (the up and the down) and a relatively heavy strange quark a possible schematic theoretical representation of the phase diagram is shown in Fig. 3 .

\subsection{Crystalline Color Superconductivity/LOFF Phase}

When considering physical applications one cannot neglect the effects of the quark masses or differences in the chemical potential among different quark flavors. If the two quarks participating in the Cooper pair posses different chemical potentials with the difference denoted by $\delta \mu$ a quark-quark bilinear condensate breaking translational and rotational invariance may emerge for certain values of $\delta \mu$ E . A similar phenomenon was suggested in the context 


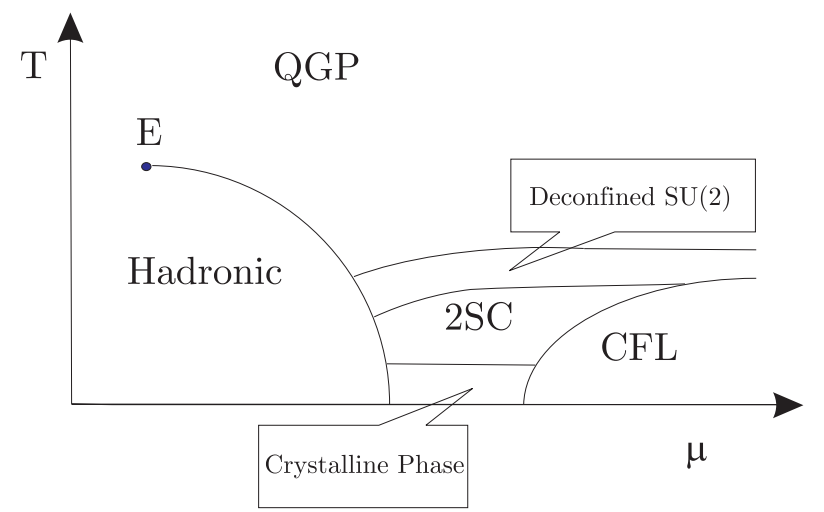

Figure 3. An oversimplified cartoon of the theoretical QCD phase diagram. The crystalline phase may exists only if we have different chemical potentials for the up and down quarks. The deconfined $S U_{c}(2)$ corresponds to the phase where we have gapped up and down quarks and the remaining unbroken $S U_{c}(2)$ of color deconfined.

of BCS theory for materials in presence of magnetic impurities by Larkin, Ovchinnikov, Fulde and Ferrel (LOFF) 27. If present in the core of neutron stars an interesting application of the LOFF phase would be the generation of glitches 26 .

\subsection{Kaon Condensation}

The effects of the strange quark mass can be quite dramatic. In the CFL phase the $K^{+}$and $K^{0}$ modes may be unstable for large values of the strange quark mass signaling the formation of a kaon condensate 28. Vortex solytions in dense quark matter due to kaon condensation have been explored in 29.

\section{$4 \quad$ Higher Spin Condensates}

At non zero quark chemical potential Lorentz invariance is explicitly broken down to the rotational subgroup $S O(3)$ and higher spin fields can condense, thus enriching the phase diagram structure of QCD and QCD-like theories. The simplest non zero spin condensate which can appear is the spin one condensate. At this point we distinguish different types of spin one condensate. 


\subsection{Superconductive Vectorial Gaps}

Rotational symmetry can break in a color superconductor if two quarks of the same flavor gap. In this case the quarks must pair in a spin one state and a careful analysis has been performed in 30 . Whether this gam occurs or not in practice is a very dynamical issue recently investigated in 31 .

\subsection{Vectorial Bose-Einstein type condensation in 2 and 3 color $Q C D$}

Differently from superconductive higher spin gaps, the vectorial Bose-Einstein type of condensation requires some, already present in the theory, composite or elementary higher spin states to couple to an external chemical potential associated to a conserved current. A simple example is the standard QCD vector field $\rho$ at non zero isospin chemical potential. It is not completely unexpected that vectors can be relevant at non zero chemical potential since they already play an important role in QCD at zero chemical potential 32 when trying to describe low energy dynamics.

Using an effective Lagrangian approach for relativistic vector fields it was shown how physical vector fields condense 33 and how the goldstone theorem is modified in this case 4 . Vector condensation for strongly interacting theories has also been suggested in 35 in the framework of two color QCD at non zero quark chemical potential where vector diquark states are present. A possible phase diagram of two color QCD for given number of light flavors augmented by a vector condensate phase is depicted in Fig. 1 .

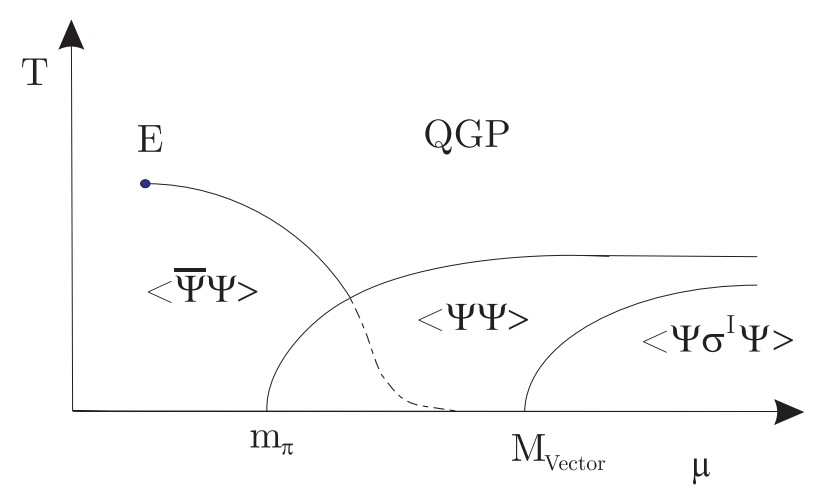

Figure 4. Schematic representation of the 2 colors phase diagram. $m_{\pi}$ is the pion mass and $M_{V e c t o r}$ is the mass of the lightest vector state. We have chosen a normalization of the baryon number such that the diquark has unit baryon number. 
In the third phase on the right we schematically represented the emergence of the vector condensate. It should coexists with the diquark condensate 35 . In this region not only we have spontaneous breaking of the baryon number but also breaking of the rotational invariance. The transition is predicted to be second order 33 .

Recent lattice simulations seem to support these predictions 36 . If these results are confirmed then for the first time we observe spontaneous rotational breaking due solely to strongly interacting matter. This fact would have far reaching consequences.

The subject of 2 color QCD at non zerg mlark chemical potential deserves a review on its own we just refer here to 37.38 .

We can already imagine a number of physical applications of topical interest. For example in the core of neutron stars or any compact object featuring a CFL state vector composite quasi-particles with masses of the order of the gap can condense due to the presence of a non zero isospin chemical potential and/or (as for kaon condensation) due to the effects of a non small strange mass. This may lead to the presence of new type of vortices affecting the compact star dynamics 29.

Interestingly vectorial Bose-Einstein condensation recently has attracted much attention in condensed matter physics since it has been observed experimentally in alkali atom gases 39 .

\section{Phases of Gauge Theories}

To discuss the QCD phase diagram as function of the number of flavors and colors at zero quark chemical potential and zero temperature we need to discuss/define in somewhat more details the phases of gauge theories.

A reasonable way to characterize the phases of gauge theories is via the potential $V(r)$ between electric test charges separated by a large distance $r$. The potential (up to a non-universal additive constant) is conjectured to behave as in the second column of Table 11. The first three phases have massless gauge fields and potentials of the form $V(r) \sim e^{2}(r) / r$. In the Coulomb phase, the electric charge $e^{2}(r)$ is constant. In the free electric phase, massless electrically charged fields renormalize the charge to zero at long distances as $e^{-2}(r) \sim \log (r \Lambda)$. Similar behavior occurs when the long distance theory is a non-Abelian theory which is not asymptotically free. The free magnetic phase occurs when there are massless magnetic monopoles, which renormalize the electric coupling constant to infinity at large distance with a conjectured behavior $e^{2}(r) \sim \log (r \Lambda)$. In the Higgs phase, the condensate of an electrically charged field gives a mass gap to the gauge fields by the Anderson-Higgs

QCDPD: submitted to World Scientific on November 16, 2018 


\begin{tabular}{|c|c|c|}
\hline Phases & Electric $V(r)$ & Magnetic $V(r)$ \\
\hline \hline Coulomb & $\frac{1}{r}$ & $\frac{1}{r}$ \\
Free Electric & $\frac{1}{r \log r \Lambda}$ & $\frac{\log r \Lambda}{r}$ \\
Free Magnetic & $\frac{\log r \Lambda}{r}$ & $\frac{1}{r \log r \Lambda}$ \\
Higgs & Constant & $\rho r$ \\
Confining & $\sigma r$ & Constant \\
\hline
\end{tabular}

Table 1. Phases of Gauge Theories

mechanism and screens electric charges, leading to a potential which, up to the additive non-universal constant, has an exponential Yukawa decay to zero at long distances. In the confining phase, there is a mass gap with electric flux confined into a thin tube, leading to the linear potential with string tension $\sigma$.

All of the above phases can be non-Abelian as well as Abelian. In particular, in addition to the familiar Abelian Coulomb phase, there are theories which have a non-Abelian Coulomb phase with massless interacting quarks and gluons exhibiting the above Coulomb potential. This phase occurs when there is a non-trivial, infrared fixed point of the renormalization group. These are thus non-trivial, interacting four dimensional conformal field theories.

It is instructive to consider the behavior of the potential $V(r)$ for magnetic test charges separated by a large distance $r$ (3th column in Table 1 ). The behavior in the first three phases can be written as $V(r)=g^{2}(r) / r$ where the effective magnetic charge $g^{2}(r)$ is related to the effective electric charge appearing by the Dirac condition, $e(r) g(r) \sim 1$. The linear potential in the Higgs phase reflects the string tension in the Meissner effect.

The above behavior is modified when there are matter fields in the fundamental representation of the gauge group because virtual pairs can pop up from the vacuum and completely screen the sources.

Note that under electric-magnetic duality, which exchanges electrically charged fields with magnetically charged fields, the behavior in the free electric phase is exchanged with that of the free magnetic phase. Mandelstam and 't Hooft suggested that the Higgs and confining phases are exchanged by duality. Confinement can then be understegd as the dual Meissner effect associated with a condensate of monopoles 40 . For supersymmetric theories

QCDPD: submitted to World Scientific on November 16, 2018 
it is possible to argue that this picture is correct 41. Dualizing a theory in the Coulomb phase, we remain in the same phase (the behavior of the potential is unchanged).

Unfortunately the previous classification of the phases of the gauge theories is not always possible. Besides if we are interested in the nature of the phase transition itself (by varying for example the temperature) the previous picture is not directly applicable. A better description/understanding can be achieved by introducing gauge invariant order parameters 42.

For theories with global symmetries we can, quite often, construct simple order parameters. This is the case of the chiral phase transition where the quark - antiquark vacuum expectation value is the order parameter and is also directly related to the hadronic states of the theory (pions, etc..). Once the order parameter is identified a mean field theory approach can be adopted. There are cases, however, where the identification of the order parameter is not trivial. And even if the order parameter is found might not be connected straightforwardly to the physical states of the theory. This is the case of the pure $S U\left(N_{c}\right)$ Yang-Mills theory where the finite temperature gauge invariant order parameter is the Polyakov loop $\ell 42,43$. Here the global symmetry group is the center $Z_{N_{c}}$ of $S U\left(N_{c}\right)$. On the other side the physical degrees of freedom of any asymptotically free gauge theory are hadronic states. Using the Yang-Mills trace anomaly and the exact $Z_{N}$ symmetry in 44 a model able to communicate to the hadrons the information carried by the order parameter was constructed.

\section{The Phase Diagram Along the Flavor Axes}

Having introduced in the previous section the correct terminology we can now investigate, at zero temperature and quark matter density, the behavior of QCD as function of number of light flavors. Besides the academic interest we also hope to use these theories to model the electroweak symmetry breaking without using an elementary higgs field 45 . Indeed the infrared behavior of gauge theories changes dramatically when changing the number of light flavors. Predictions resulting from exact theoreticaltreatments are, at the moment, only possible for supersymmetric theories 41 . Nevertheless for non supersymmetric QCD theories it is still possible, using different methods and models, to sketch a plausible phase diagram which can be tested via lattice simulations. We focus here on zero quark chemical potential and temperature. We will however suggest how the dynamics of large number of flavors QCD might influence any other phase. 


\subsection{The Coulomb/Conformal Phase}

For $N_{f}>\frac{11}{2} N_{c}$, the one-loop beta function of QCD changes sign and the theory loses asymptotic freedom. The resulting infrared free theory is now in a non-Abelian QED-like phase in which neither confinement nor chiral symmetry breaking is expected. For values of $N_{f}$ near but below $\frac{11}{2} N_{c}$, the beta function develops a perturbative infrared stable fixed point at which the trace of the energy momentum tensor vanishes exactly and the theory is a nonAbelian conformal field theory (Coulomb phase). In this phase, the coupling constant is small on account of the large number of flavors and so we do not expect any of the global symmetries to break. However, as the number of flavors is decreased, the fixed point becomes nonperturbative and the coupling constant increases to a critical value above which chiral symmetry breaks spontaneously. A dynamical scale is generated and conformal symmetry is lost. The generation of this scale defines the critical number of massless flavors, i.e. the minimum number of flavors for which the gauge theory is still conformal and chiral symmetry is still intact. Below this critical number of flavors, the theory is expected to confine and the low energy spectrum is hadronic. This discussion assumes that the conformal and chiral phase transitions coincide as function of the number of flavors, but whether or not this is true is still controversial. This is not the case for supersymmetric QCD (see right panel in Fig. 5) where for a certain range of flavors the global symmetries do not break but the theory is still assumed to confine We will assume here, as corroborated by lattice simulations for $N_{c}=346$ (though more investigations are needed), that there is in fact a single conformal/chiral phase transition. Figure 5 (left panel) summarizes the possible phase structure for QCD as a function of the number of light flavors.

\subsection{Conformal Critical Exponents}

By way of a simple model it is possible to describe the conformal/chiral phase transition 47 . Since chiral symmetry, in the present scenario, is linked to the breaking of the conformal symmetry the model uses the chiral condensate $\sigma \sim \bar{q} q$ as the order parameter. Trace and axial anomaly were also used to constrain part of the potential. In the absence of the quark masses the condensate vanishes exponentially fast as $N_{f}$ approaches the critical value. More precisely the following result for the physical mass $M_{\sigma}$ and $\langle\sigma\rangle$ was derived in 4 :

$$
<\sigma>\simeq\left[\frac{\gamma-1}{2|C|}\right]^{\frac{1}{2(\gamma-1)}} \Lambda, \quad M_{\sigma} \simeq 2 \sqrt{6}|C|\left[\frac{\gamma-1}{2|C|}\right]^{\frac{1}{2(\gamma-1)}} \Lambda .
$$

QCDPD: submitted to World Scientific on November 16, 2018 
Likewise, in the presence of the quark mass term we have

$$
[<\sigma>]_{\gamma=1} \simeq\left[\frac{m N_{f} \Lambda}{2|C|}\right]^{\frac{1}{2}}, \quad\left[M_{\sigma}\right]_{\gamma=1} \simeq 2\left[2 m N_{f} \Lambda\right]^{\frac{1}{2}} .
$$

Here $\gamma \geq 1$ (in the confined and highly non perturbative phase) is the anomalous dimension of the quark mass operator and $C$ an unknown coefficient. Thus the order parameter $\sigma$ for $\gamma=1$ vanishes according to the power $1 / 2$ with the quark mass in contrast with an ordinary second order phase transition where the order parameter is expected to vanish according to the power $1 / 3$. We expect that for a vanishing beta function close to the conformal point the theory becomes conformal at $\gamma=1$. This fixes the critical value of the number of flavors to $4 N_{c}$. For more details and a complete list of references we refer the reader to the original paper 4 . Lattice simulations would certainly provide very useful in understanding the nature of this conformal phase and associated phase transition features.

\subsection{Enhanced Symmetries Scenario}

When the number of flavors is just below the critical value, the theory still exhibits chiral symmetry breaking but is possible that the vector spectrum changes quite significantly. In Refs. 496 it was suggested that a new global symmetry may be dynamically generated. This symmetry acts on the massive spectrum of the theory and is related to the modification of the second Weinberg spectral function sum rule near the critical number of flavors 5 .

If such an enhanced symmetry emerges, the vectors along the broken generators become degenerate with those along the orthogonal directions. For $N_{c}=3 \mathrm{QCD}$, this corresponds to mass degenerate vector ( $\rho$-type of field) and axial particles even in the presence of chiral symmetry breaking.

In the enhanced symmetry scenario, the interactions between the vectors and the Goldstone excitations are suppressed (in the derivative expansion). The enhanced symmetry scenario imposes very stringent constraints on the possible form of the negative intrinsic parity terms (the ones involving the $\epsilon_{\mu \nu \rho \sigma}$-terms) as well to a

We conjecture the following phase structure before entering the conformal phase:

\footnotetext{
${ }^{a}$ Nota Bene: In reference 18 the gauging of the Wess-Zumino term needed for the saturation of the ' $t$ Hooft global anomaly matching has been computed for 2-colors QCD. The effective theories for 2 color QCD at zero density for the linear and non linear realization extended, as well, to incl the Electroweak theory and to contain physical vectors states were first constructed in 49 . 4 for an arbitrary number of flavors.
} 
- Approximate local chiral symmetry for small $N_{f}$

- Parity doubling and an extra global symmetry near the critical $N_{f}$.

At very low number of flavors the vectors can be included in the low energy effective theory as almost gauge vectors of the chiral symmetry. By "almost" we mean that the vector masses are considered as minimal sources of breaking of the local chiral symmetry. This hypothesis is at the base of "vector dominance" model, it strongly reduces the number of unknown coefficients in the effective Lagrangian and it has been widely used in the past for successful phenomenological investigations. Near the critical $N_{f}$, according to our conjecture, a new dynamically generated global symmetry sets in. Due to this new symmetry the vectors are almost degenerate while chiral symmetry still breaks spontaneously. In this regime the new global symmetry strongly constrains the effective Lagrangian theory.

Interestingly enough when extending this theory to model the spontaneous symmetry breaking sector of the electroweak theory the major contribution to the $S$-parameter (proportional schematically to the difference between the square of the mass of the axial vector and vector $M_{A}^{2}-M_{V}^{2}$ ) is protected by the new enhanced symmetry and leads to phenomenologically viable technicolor models of the type of the BESS models 5 .

For a fixed, nonzero chemical potential, the phase structure as the number of light flavors is increased should be very rich 52. For instance, when $N_{f}>$ $\frac{11}{2} N_{c}$, the theory is no longer asymptotically free and the low energy theory is simply the QCD Lagrangian. In this regime perturbation theory is reliable also at low energy scales.

\section{Conclusions}

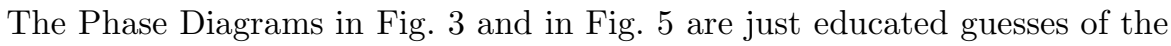
true QCD phase diagram. Indeed other interesting phases may emerge in the future recalling the richness of the phases encountered in other physical systems studied in the condensed matter framework.

\section{Acknowledgments}

It is a pleasure to thank P. Damgaard, A.D. Jackson, K. Rummukainen, J. Schechter, W. Schäfer and K. Splittorff for discussions and careful reading of the manuscript. I also thank R. Casalbuoni, J.T. Lenaghan, M.P. Lombardo and G. Nardulli for useful discussions. This work is supported by the Marie-Curie fellowship under contract MCFI-2001-00181.

QCDPD: submitted to World Scientific on November 16, 2018 


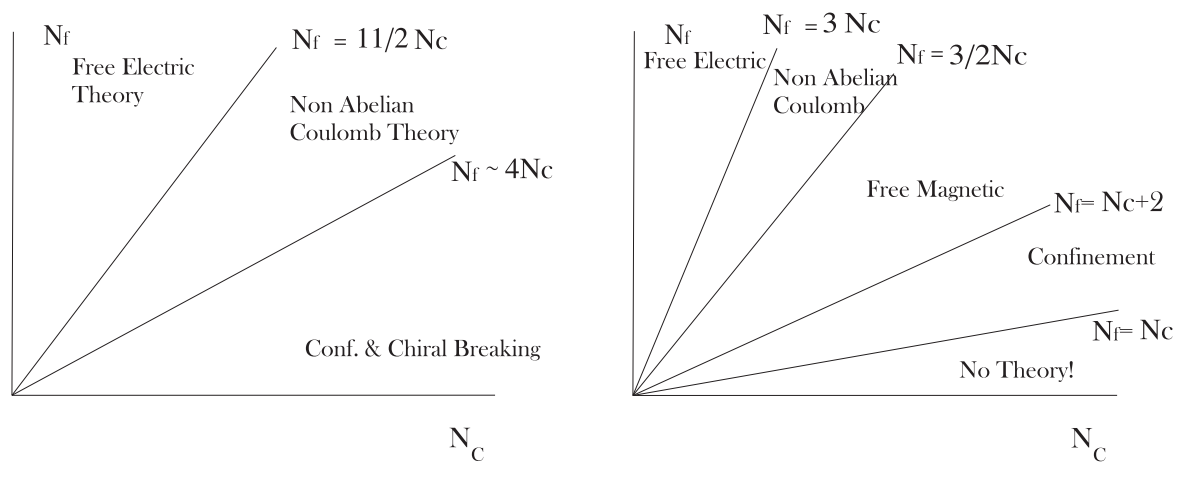

Figure 5. Left Panel: Represent a possible plan for the QCD phase diagram at zero quark density and temperature but for different number of flavors and colors. The origin in both schemes of the number of color axes corresponds to $N_{c}=2$. Right Panel: Same phase diagram for supersymmetric QCD.

\section{References}

1. See K. Rajagopal and F. Wilczek, hep-ph/0011333; M.G. Alford, hep$\mathrm{ph} / 0102047$ for an overview on the subject; S.D.H. Hsu, hep-ph/0003140 for the renormalization group approach review; D.K. Hong, Acta Phys. Polon. B32:1253, 2001, hep-ph/0101025 for the effective theories close to the fermi surface.

2. H. Satz, arXiv:hep-ph/0201051.

3. E. V. Shuryak, arXiv:hep-ph/0110130.

4. M. A. Halasz, A. D. Jackson, R. E. Shrock, M. A. Stephanov and J. J. Verbaarschot, Phys. Rev. D 58, 096007 (1998) arXiv:hep-ph/9804290].

5. M. P. Lombardo, arXiv:hep-ph/0103141.

6. R. Casalbuoni, hep-th/0108195/ for the effective Lagrangians approach.

7. G. Nardulli, hep-ph/0202037 for the effective theory approach to CSC and the LOFF phase and possible applications of the LOFF phase to the physics of compact stars.

8. U. W. Heinz, AIP Conf. Proc. 602, 281 (2001) arXiv:hep-ph/0109006.

9. G. Boyd, J. Engels, F. Karsch, E. Laermann, C. Legeland, M. Lutgemeier and B. Petersson, Nucl. Phys. B 469, 419 (1996) arXiv:hep-lat/9602007.

10. M. Okamoto et al. [CP-PACS Collaboration], Phys. Rev. D 60, 094510 (1999) arXiv:hep-lat/9905005.

11. Z. Fodor and S. D. Katz, arXiv:hep-lat/0204029.

12. G. W. Carter and S. Reddy, Phys. Rev. D 62, 103002 (2000), hep-

QCDPD: submitted to World Scientific on November 16, 2018 
$\mathrm{ph} / 0005228$.

13. D. K. Hong, S. D. Hsu and F. Sannino, Phys. Lett. B 516, 362 (2001), hep-ph/0107017.

14. R. Ouyed and F. Sannino, astro-ph/0103022. To appear in Astronomy \& Astrophysics.

15. R. Casalbuoni and R. Gatto, Phys. Lett. B464, 11 (1999).

16. F. Sannino, Phys. Lett. B480, 280, (2000). S. D. Hsu, F. Sannino and M. Schwetz, Mod. Phys. Lett. A 16, 1871 (2001), hep-ph/0006059.

17. R. Casalbuoni, Z. Duan and F. Sannino, Phys. Rev. D 62 (2000) 094004, hep-ph/0004207.

18. R. Casalbuoni, Z. Duan and F. Sannino, Phys. Rev. D 63, 114026 (2001), hep-ph/0011394

19. D. H. Rischke, D. T. Son and M. A. Stephanov, Phys. Rev. Lett. 87, 062001 (2001), hep-ph/0011379.

20. R. Ouyed and F. Sannino, Phys. Lett. B 511, 66 (2001).

21. F. Sannino, N. Marchal and W. Schafer, arXiv:hep-ph/0202248.

22. R.D. Pisarski and D.H. Rischke, Phys. Rev. D61, 051501 (2000); Phys. Rev. D61, 074017 (2000).

23. D. K. Hong and S. D. Hsu, arXiv:hep-ph/0202236.

24. D. F. Litim and C. Manuel, Phys. Rev. Lett. 87, 052002 (2001), hep$\mathrm{ph} / 0103092$.

25. M. Alford and K. Rajagopal, arXiv:hep-ph/0204001.

26. J. A. Bowers and K. Rajagopal, arXiv:hep-ph/0204079 and references therein.

27. A.I. Larkin and Yu.N. Ovchinnikov, Zh. Eksp. Teor.Fiz., 47, (1964) 1136 (Sov. Phys. JETP, 20, (1965) 762); P. Fulde and R.A. Ferrell, Phys. Rev. 135, (1964) A550.

28. T. Schafer, Phys. Rev. Lett. 85, 5531 (2000) arXiv:nucl-th/0007021. T. Schafer, D. T. Son, M. A. Stephanov, D. Toublan and J. J. Verbaarschot, Phys. Lett. B 522, 67 (2001) arXiv:hep-ph/0108210. D. B. Kaplan and S. Reddy, Phys. Rev. D 65, 054042 (2002) arXiv:hep$\mathrm{ph} / 0107265$.

29. D. B. Kaplan and S. Reddy, Phys. Rev. Lett. 88, 132302 (2002).

30. R. D. Pisarski and D. H. Rischke, Phys. Rev. D 61, 074017 (2000) arXiv:nucl-th/9910056.

31. M. Buballa, J. Hosek and M. Oertel, arXiv:hep-ph/0204275.

32. M. Harada, F. Sannino and J. Schechter, Phys. Rev. D 54, 1991 (1996) arXiv:hep-ph/9511335. M. Harada, F. Sannino and J. Schechter, Phys. Rev. Lett. 78, 1603 (1997), [arXic:|hep-ph/9609428].

33. F. Sannino and W. Schäfer, Phys. Lett. B 527, 142 (2002) hep-

QCDPD: submitted to World Scientific on November 16, 2018 
ph/0111098; F. Sannino and W. Schäfer. These proceedings hep$\mathrm{ph} / 0204353$.

34. H.B. Nielsen and S. Chadha, Nucl. Phys. B105, 445 (1976). V. A. Miransky and I. A. Shovkovy, Phys. Rev. Lett. 88, 111601 (2002) arXiv:hep$\mathrm{ph} / 0108178$.

35. J. T. Lenaghan, F. Sannino and K. Splittorff, Phys. Rev. D 65, 054002 (2002) arXiv:hep-ph/0107099.

36. M.P. Lombardo, these proceedings.

37. J. B. Kogut, D. K. Sinclair, S. J. Hands and S. E. Morrison, Phys. Rev. D 64, 094505 (2001) arXiv:hep-lat/0105026; I. Montvay, S. Hands, L. Scorzato and J. I. Skullerud, arXiv:hep-lat/0112046.

38. J.B. Kogut, M.A. Stephanov, and D. Toublan, Phys. Lett. B 464 (1999) 183; J.B. Kogut, M.A. Stephanov, D. Toublan, J.J.M. Verbaarschot, and A. Zhitnitsky, Nucl.Phys. B 582 (2000) 477; K. Splittorff, D. Toublan, and J.J.M. Verbaarschot, Nucl. Phys. B620, 290 (2002) arXiv:hepph/0108040, arXiv:hep-ph/0204076.

39. D.M. Stamper-Kurn, M.R. Andrews, A.P. Chikkatur, S. Inouye, H.J. Miesner, J. Stenger, and W. Ketterle, Phys. Rev. Lett. 80, 2027 (1998).

40. A. D. Giacomo, arXiv:hep-lat/0204001.

41. K. Intriligator and N. Seiberg, Nucl. Phys. Proc. Suppl. 45BC, 1 (1996).

42. B. Svetitsky and L. G. Yaffe, Nucl. Phys. B 210, 423 (1982). L. G. Yaffe and B. Svetitsky, Phys. Rev. D 26, 963 (1982). B. Svetitsky, Phys. Rept. 132, 1 (1986).

43. R. D. Pisarski, hep-ph/0112037; R.D. Pisarski, Phys. Rev. D62, 111501 (2000).

44. F. Sannino, arXiv:hep-ph/0204174.

45. K. Lane, arXiv:hep-ph/0202255.

46. R. D. Mawhinney, Nucl. Phys. Proc. Suppl. 60A, 306 (1998), heplat/9705030; D. Chen, R. D. Mawhinney, Nucl. Phys. Proc. Suppl. 53, 216 (1997).

47. F. Sannino and J. Schechter, Phys. Rev. D60, 056004, (1999).

48. Z. Duan, P.S. Rodrigues da Silva and F. Sannino, Nucl. Phys. B 592, 371 (2001), hep-ph/0001303.

49. T. Appelquist, P.S. Rodrigues da Silva and F. Sannino, Phys. Rev. D60, 116007 (1999), hep-ph/9906555.

50. T. Appelquist and F. Sannino, Phys. Rev. D59, 067702 (1999).

51. R. Casalbuoni, S. De Curtis and M. Redi, Eur. Phys. J. C 18, 65 (2000) arXiv:hep-ph/0007097.

52. T. Schäfer, Nucl. Phys. B 575, 269 (2000) arXiv:hep-ph/9909574.

QCDPD: submitted to World Scientific on November 16, 2018 